J. Westerbacka $\cdot$ E. Leinonen $\cdot$ J. T. Salonen •

R. Salonen · A. Hiukka · H. Yki-Järvinen ·

M.-R. Taskinen

\title{
Increased augmentation of central blood pressure is associated with increases in carotid intima-media thickness in type 2 diabetic patients
}

Received: 13 July 2004 / Accepted: 26 February 2005 / Published online: 23 June 2005

(C) Springer-Verlag 2005

\begin{abstract}
Aims/hypothesis: Type 2 diabetes is associated with a two- to seven-fold increase in cardiovascular morbidity and mortality. The aim of this study was to determine the relationships between intima-media thickness (IMT), an established marker of atherosclerosis, large artery function and other determinants of cardiovascular risk in type 2 diabetic patients. Methods: We studied 228 type 2 diabetic patients $(75$ women, aged $62 \pm 2$ years [mean \pm SEM]). Carotid IMT was bilaterally measured using ultrasound technology. Applanation tonometry and pulse wave analysis were used to measure aortic systolic and diastolic blood pressures, central pressure augmentation (AG) and the augmentation index (AIx), a measure of systemic arterial stiffness. Conventional cardiovascular risk factors (lipids, $\mathrm{HbA}_{1} \mathrm{c}$, smoking and diabetes duration) were also assessed. Results: Women had higher AG and AIx $(p<0.0001)$, despite comparable systolic BP and heart rate in women and men. In women, $\mathrm{AG}(r=0.39, p<0.001)$, age $(r=0.32, p<0.01)$, brachial systolic BP $(r=0.34, p<$ $0.01)$ and aortic systolic BP $(r=0.34, p<0.01)$ correlated with IMT. In men, age $(r=0.41, p<0.001)$, diabetes duration $(r=0.25, p<0.01)$, AG $(r=0.22, p<0.01)$, aortic sys-
\end{abstract}

J. Westerbacka · E. Leinonen · A. Hiukka

H. Yki-Järvinen · M.-R. Taskinen

Department of Medicine,

University of Helsinki,

Helsinki, Finland

J. T. Salonen · R. Salonen

The Research Institute of Public Health,

University of Kuopio,

Kuopio, Finland

J. T. Salonen

Oy Jurilab,

Kuopio, Finland

J. Westerbacka $(\square)$

Department of Medicine, Biomedicum,

P.O. Box 700, Haartmaninkatu 8,

00029 HUCH Helsinki, Finland

e-mail: jukka.westerbacka@helsinki.fi

Tel.: +358-9-47171885

Fax: +358-9-47175585 tolic BP $(r=0.21, p<0.01)$, brachial systolic BP $(r=0.21$, $p<0.01)$ and body weight $(r=0.16, p<0.05)$ correlated with IMT. In multiple linear regression analyses, AG and aortic systolic BP, but not brachial systolic BP, were age-independent determinants of IMT in men and women. In all patients, increased AG (adjusted for sex, age and heart rate) correlated with longer duration of diabetes, urinary albumin excretion and IMT. Conclusions/interpretation: Measures of central systolic pressure correlate with carotid IMT, independently of age and other risk markers.

Keywords Arterial stiffness - Intima-media thickness · Lipoproteins - Pulse wave analysis - Type 2 diabetes . Ultrasound · Wave reflection

Abbreviations AG: Central pressure augmentation . AIx: Augmentation index - CVD: cardiovascular disease . FIELD: Fenofibrate Intervention and Event Lowering in Diabetes - IMT: intima-media thickness - PWA: pulse wave analysis

\section{Introduction}

Type 2 diabetes is associated with a two- to seven-fold increased risk of cardiovascular disease [1-3]. The excessive risk is not explained by classic risk factors (LDL cholesterol, smoking and brachial blood pressure) [2]. In a recent analysis of the Hoorn Study, ultrasonically measured peripheral arterial stiffness increased in parallel with worsening glucose tolerance in elderly subjects [4] independently of classic risk factors, hyperglycaemia or hyperinsulinaemia.

Several studies have documented a close correlation between cardiovascular disease and arterial stiffening in non-diabetic patients using a variety of techniques including ultrasound [5-7], magnetic resonance imaging with cine velocity mapping [8], and pulse wave velocity $[9,10]$. In contrast to these relatively time-consuming and operator-dependent methods, the non-invasive technique of applanation tonometry can record peripheral arterial pressure 
waveforms and accurately generate aortic pressure waveforms using a validated transfer function within a few minutes $[11,12]$. Central pressure augmentation $(\mathrm{AG})$, i.e. the pressure difference between the second (reflected) and first systolic peaks, can also be measured from the aortic pressure waveform. Arterial stiffening increases pulse wave velocity, which results in the earlier return of the reflected wave. This increases augmentation and central systolic pressure [13]. The augmentation index (AIx) is calculated by dividing augmentation by pulse pressure. AG and AIx provide measures of wave reflections and global arterial stiffness, and are increased by cardiovascular risk factors such as age [13, 14], smoking [15-17] and hypertension $[18,19]$. Central pulse pressure [20] and AIx [21] were recently shown to predict cardiovascular mortality more accurately than pulse wave velocity in a prospective study of patients with end-stage renal failure and a markedly increased risk of cardiovascular mortality [22]. Recently, in men undergoing coronary angiography, AG and AIx were found to be independent markers of the severity of coronary artery disease [23].

High-resolution B-mode ultrasonography is a non-invasive technique used to measure the intima-media thickness (IMT) of the arterial wall [24-26]. It is a validated surrogate marker of atherosclerosis [27]. Carotid IMT and coronary artery disease events are positively correlated [28, 29] and carotid IMT predicts cardiovascular mortality $[30,31]$. Brachial artery blood pressure does not reflect central blood pressure [13, 32], which on the other hand is the pressure affecting the heart and carotid arteries. One could therefore expect aortic systolic pressure, which determines AG, to be correlated with IMT in the carotid artery. If so, measurement of AG and AIx might help to assess cardiovascular risk. In the present study, we investigated whether AG or AIx are associated with IMT in type 2 diabetic patients. We were particularly interested to establish whether AG or AIx correlates with IMT more closely than brachial artery blood pressure, and how these measures are related to other cardiovascular risk factors. As a secondary aim, we examined the association of local soft tissue and mineralised arterial wall changes with AG and AIx in a subgroup of 97 patients, since these structural changes may provide additional information about atherosclerosis beyond IMT $[33,34]$.

\section{Subjects and methods}

Study subjects Study subjects were type 2 diabetic patients participating in the FIELD (Fenofibrate Intervention and Event Lowering in Diabetes) Study at the Helsinki Centre, Finland. The FIELD Study is an ongoing multinational, double-blind study taking place in Australia, New Zealand and Finland. Altogether, over 9,000 type 2 diabetic patients are participating in the study. The patients have been randomly assigned to receive either placebo or micronised fenofibrate $(200 \mathrm{mg} /$ day $)$ for 5 years. Type 2 diabetic subjects between 50 and 75 years of age, with serum cholesterol values between 1.0 and $5.5 \mathrm{mmol} / \mathrm{l}$ and either serum triglycerides between 1.0 and $5.0 \mathrm{mmol} / \mathrm{l}$ or a cholesterol : HDL cholesterol ratio of above 4, were eligible to take part. Subjects with hepatic or renal dysfunction, gallstones, lipid-lowering medication, cyclosporin, alcohol overuse or other severe mental or physical illness were not eligible to participate.

At the Helsinki Centre, 270 patients were recruited for the FIELD main study. Of these, 228 (76 female) participants volunteered to take part in this substudy. All biochemical analyses, IMT and pulse wave analysis (PWA) measurements were done before randomisation. Of the substudy participants, 71 were diagnosed as having cardiovascular disease (CVD, defined as the presence or previous occurrence of one or more of the following: angina pectoris, coronary bypass graft or balloon dilatation, stroke, transient ischaemic attack, carotid endarterectomy, claudication, leg amputation, peripheral arterial reconstruction or balloon dilatation); 116 patients were hypertensive as defined by a resting blood pressure of above 140/90 $\mathrm{mmHg}$ or use of antihypertensive medication.

Each subject gave their written informed consent prior to participating in the study. The study was approved by the ethics committee of the Helsinki University Central Hospital. All the samples were collected in accordance with the Helsinki declaration.

Biochemical analyses Venous blood samples for the biochemical measurements were obtained in the morning after an overnight fast. All laboratory samples were obtained during the placebo run-in phase of the FIELD Study before fenofibrate intervention. Serum and EDTA plasma were separated by centrifugation and stored at $-80^{\circ} \mathrm{C}$ until analysed. All lipid measurements were performed in the research laboratory of the Helsinki University Central Hospital (Division of Cardiology, Helsinki, Finland). Serum total cholesterol and triglycerides were determined by enzymatic methods (kits 0722138 and 0715166 respectively; Hoffman-La Roche; Basle, Switzerland) using an automated Cobas Mira analyser (Hoffman-La Roche). Serum HDL cholesterol was quantified by the phosphotungastic acid/magnesium chloride precipitation procedure (HoffmanLa Roche kit 0720674). LDL cholesterol was calculated using the formula developed by Friedewald. Plasma glucose concentrations were measured by a glucose dehydrogenase method (Precision-G blood glucose testing system, Medisense; Abbott, Espoo, Finland). Glycosylated haemoglobin $\left(\mathrm{HbA}_{1} \mathrm{c}\right)$ was measured using a commercially available kit (DCA 2000 Analyzer; Bayer Diagnostics, Pittsburgh, PA, USA). The albumin excretion rate was measured from three subsequent overnight urinary collections.

Anthropometric variables Body weight and height were measured and BMI was calculated as $\mathrm{kg} / \mathrm{m}^{2}$. Waist and hip circumferences were also recorded and the waist-to-hip ratio was used as a measure of body fat distribution. 
Carotid sonography Ultrasound scanning of carotid arteries was performed using a Hewlett-Packard Image Point M2410A ultrasound system equipped with a high-frequency $10-\mathrm{MHz}$ linear array transducer. Scans were videotaped using a Panasonic AG-MD830E PAL S-VHS video cassette recorder. All ultrasound examinations were carried out by the same physician (E. Leinonen). The ultrasound examination protocol has previously been described in detail [35]. A single reader at Oy Jurilab (Kuopio, Finland; http://www.jurilab.com) took the IMT measurements using a PC with a video frame grabber interfaced to a PAL S-VHS video cassette recorder. The Prosound software was used to measure the IMTs at a total of 28 sites. The Prosound software allows 100 measurements per $\mathrm{cm}$ of edge length. IMT is calculated as the mean difference between the intima/lumen and media/adventitia interfaces from the 100 measurements. The following variables were derived for each site: the mean IMT, the maximum IMT and the minimum IMT. All outcome variables were first calculated for each subject. The mean of the maximum IMT measurements over 28 sites (hereafter IMT) was chosen for the primary outcome variable. Secondary outcome variables were as follows: (1) the mean of the mean IMT measurements over 28 sites (mean IMT); (2) the mean far wall IMT; (3) the mean of the maximum IMT measurements for the common carotid artery; (4) the mean of the maximum IMT measurements for the carotid bulb; and (5) the mean of maximum for the internal carotid artery.

Scoring of structural changes In 97 randomly selected type 2 diabetic patients, the ultrasound scans were further scored according to local soft tissue and mineralisation changes at all carotid sites. The scoring system for soft changes was as follows: $0=$ no change; $1=$ thickening by $1.5 \mathrm{~mm}$ or more in the common carotid artery or $2.0 \mathrm{~mm}$ or more in the internal carotid artery or carotid bulb; $2=$ protrusion of $25-39 \%$ of lumen diameter; $3=$ protrusion of $40 \%$ or more of lumen diameter. The scoring system for mineralisation changes was as follows: $0=$ no mineralisation; $1=$ few scattered changes; $2=$ several changes or mineralisation cluster. The maximum soft change and mineralisation score for each patient was used for analysis.

Pulse wave analysis The technique of pulse wave analysis (PWA) was used to determine central aortic pressure, AG and AIx as previously described in detail [36]. All measurements were taken from the radial artery by applanation tonometry using a Millar tonometer (SPC-301; Millar Instruments, Houston, TX, USA). Procedures were performed by a single investigator (J. Westerbacka) blinded for ultrasound measurements. Collected data were loaded directly into a desktop computer and processed by the SphygmoCor blood pressure analysis system (BPAS-1; AtCor Medical, Sydney, Australia), which allows continuous online recording of the radial artery pressure waveform. The integral system software was used to calculate an average radial artery waveform and to generate the corresponding ascending aortic pressure waveform using a previously validated transfer factor $[12,37]$. The AIx was calculated by dividing AG by pulse pressure $[10,14]$. Blood pressure was measured using a calibrated mercury sphygmomanometer after the subject had rested in the supine position for $10 \mathrm{~min}$. The measurements were performed after an overnight fast and the patients did not take any medication in the morning prior to the measurements.

Statistical analyses Statistical comparisons of clinical and biomedical parameters were performed using the SPSS statistical package (version 11.0; SPSS, Chicago, IL, USA). The data were initially analysed separately in men and women and were then confirmed in the whole study group. Data are presented as means \pm SEM for continuous variables and as frequencies or percentages for categorical variables. Variables with non-normal distribution were $\log _{10}$ transformed to approximate Gaussian distribution and homogeneous variances. Results from lipid and lipoprotein determinations, IMT and PWA measurements and other continuous variables were initially compared between women and men using Student's $t$-test. The frequency distributions of the categorical variables in the two groups were compared by the chi square test. Secondly, univariate correlations for IMT were analysed separately in men and women. Thirdly, multivariate models (including significant correlates from univariate analyses) were employed to investigate the importance of individual variables. Finally, the correlates for IMT and augmentation were confirmed in the whole study group after adjustment for age and sex (both affecting IMT and augmentation) and heart rate (affecting central wave reflections and therefore augmentation [38]). The differences between the subgroup and the whole group were analysed using Student's $t$-test or the chi square test as appropriate. The differences across the soft and mineralisation categories were analysed using one-way ANOVA followed by Bonferroni's post-hoc test. For soft change categories, categories 2 and 3 were combined due to a low number of subjects in the score 3 category. A $p$ value of less than 0.05 was considered statistically significant.

\section{Results}

Subject characteristics A total of 228 type 2 diabetic patients ( 75 women) participated in the study. Clinical and biochemical characteristics of the study subjects are presented in Table 1. Male and female type 2 diabetic patients did not differ with respect to age, duration of diabetes or BMI. As expected, women had higher serum HDL cholesterol and lower waist-to-hip ratio than men. There were more ex- and current smokers in women than men. The prevalence of hypertension and coronary heart disease was similar in men and women. The type 2 diabetic patients randomly selected for arteriosclerosis scoring analysis did not differ from the whole diabetic group with respect to age, sex, BMI, blood pressure, $\mathrm{HbA}_{1} \mathrm{c}$, diabetes duration or prevalence of hypertension or CHD (data not shown). 
Table 1 Characteristics of the type 2 diabetic patients

\begin{tabular}{lccc}
\hline & All & Men & Women \\
\hline Number & 228 & 153 & 75 \\
Age (years) & $62 \pm 1$ & $62 \pm 1$ & $62 \pm 1$ \\
Duration of diabetes (years) & $8 \pm 1$ & $8 \pm 1$ & $8 \pm 1$ \\
Body mass index (kg/m $\left.{ }^{2}\right)$ & $31 \pm 1$ & $31 \pm 1$ & $30 \pm 1$ \\
Waist-to-hip ratio & $0.93 \pm 0.01$ & $0.96 \pm 0.01$ & $0.88 \pm 0.01 * * *$ \\
fS total cholesterol (mmol/l) & $5.1 \pm 0.1$ & $5.0 \pm 0.1$ & $5.2 \pm 0.1$ \\
fS LDL cholesterol (mmol/l) & $3.2 \pm 0.1$ & $3.2 \pm 0.1$ & $3.2 \pm 0.1$ \\
fS HDL cholesterol (mmol/l) & $1.2 \pm 0.1$ & $1.1 \pm 0.1$ & $1.4 \pm 0.1 * * *$ \\
fS triglycerides (mmol/l) & $1.8 \pm 0.1$ & $1.7 \pm 0.1$ & $1.8 \pm 0.1$ \\
fS glucose (mmol/l) & $7.9 \pm 0.1$ & $7.8 \pm 0.2$ & $8.0 \pm 0.1$ \\
HbA 1 c (\%) & $7.3 \pm 0.1$ & $7.2 \pm 0.1$ & $7.4 \pm 0.2$ \\
Smoking (never/ex/current) & $85 / 109 / 34$ & $75 / 57 / 21$ & $10 / 52 / 13 * * *$ \\
\hline
\end{tabular}

$f S$ fasting serum; $\mathrm{Hb}_{1} \mathrm{c}$ glycosylated haemoglobin (normal range 4-6\%)

$* * * p<0.001$ for men vs women

Data are shown as means \pm SEM

IMT and PWA in women and men The results of the IMT and PWA measurements are shown in Table 2. All IMT measurements, except the IMT of the common carotid artery, were significantly higher in men than in women. Both brachial and aortic systolic and mean blood pressures were similar in men and women. However, in women, diastolic blood pressure was lower and pulse pressure was higher than in men in both the brachial artery and the aorta. Although heart rate was similar in women and men, both AG and AIx were higher in women than in men. After adjustment for height and smoking, which may affect the wave reflections and therefore AG, women still had higher AG $(p<0.05)$ and AIx $(p<0.05)$ than men. The
AG and AIx were comparable in diabetic patients with clinical cardiovascular disease and those without (data not shown).

Correlates of carotid IMT in women and men In women, following simple regression analysis, $\mathrm{AG}(r=0.39, p<$ $0.001)$, age $(r=0.32, p<0.01)$, brachial systolic BP $(r=0.34$, $p<0.01)$ and aortic systolic BP $(r=0.34, p<0.01)$ correlated with IMT. Brachial diastolic BP $(r=-0.05$, NS), aortic diastolic BP ( $r=-0.03$, NS), weight, BMI, smoking, $\mathrm{HbA}_{1} \mathrm{c}$, diabetes duration and lipids did not correlate with IMT. Following multiple linear regression analysis $\left(R^{2}=24.9 \%\right.$, $p<0.001)$, AG $(p<0.02)$ and aortic systolic BP $(p<0.05)$, but not brachial systolic BP, were age-independent $(p<$ 0.001 ) determinants of IMT in women.

In men, following simple regression analysis, age ( $r=$ $0.41, p<0.001)$, diabetes duration $(r=0.25, p<0.01)$, AG $(r=0.22, p<0.01)$, aortic systolic BP $(r=0.21, p<0.01)$, brachial systolic BP $(r=0.21, p<0.01)$ and body weight $(r=0.16, p<0.05)$ correlated with IMT, whereas brachial diastolic BP $(r=-0.06, \mathrm{NS})$, aortic diastolic BP $(r=-0.04$, $\mathrm{NS}$ ), smoking, $\mathrm{HbA}_{1} \mathrm{c}$ and lipids did not. Following multiple linear regression analysis $\left(R^{2}=27.8 \%, p<0.001\right)$, AG $(p<0.05)$ and aortic systolic BP $(p<0.05)$, but not brachial systolic BP, significantly explained variation in IMT independently of age $(p<0.001)$ and weight $(p<0.01)$ in men.

Correlates of $A G$ and IMT in the whole study group In the whole study group (Table 3), AG (adjusted for sex, age and heart rate) correlated with duration of diabetes, urinary albumin excretion rate and IMT. In a similar analysis, IMT (adjusted for sex and age) correlated with urinary albumin excretion rate, AG and AIx (Table 3).
Table 2 Intima-media thickness (IMT) and haemodynamic parameters measured by pulse wave analysis in type 2 diabetic patients

Maximum IMT, mean of maximum IMT

${ }^{*} p<0.05,{ }^{* *} p<0.01, * * * p<0.001$

for men vs women

Data are means \pm SEM

\begin{tabular}{lrrc}
\hline & \multicolumn{1}{l}{ All } & \multicolumn{1}{l}{ Men } & Women \\
\hline IMT & & & \\
Maximum IMT (mm) & $1.33 \pm 0.02$ & $1.36 \pm 0.02$ & $1.27 \pm 0.02^{* * * *}$ \\
Mean IMT (mm) & $1.05 \pm 0.01$ & $1.07 \pm 0.02$ & $1.01 \pm 0.02^{* * *}$ \\
Far wall IMT (mm) & $1.05 \pm 0.01$ & $1.071 \pm 0.02$ & $1.01 \pm 0.01^{* *}$ \\
Common carotid artery IMT (mm) & $1.19 \pm 0.01$ & $1.20 \pm 0.01$ & $1.17 \pm 0.02$ \\
Carotid bulb IMT (mm) & $1.51 \pm 0.02$ & $1.54 \pm 0.03$ & $1.44 \pm 0.03^{*}$ \\
Internal carotid artery IMT (mm) & $1.22 \pm 0.03$ & $1.27 \pm 0.03$ & $1.12 \pm 0.04^{* * *}$ \\
Pulse wave analysis & & & \\
Brachial systolic BP (mmHg) & $148 \pm 1$ & $147 \pm 2$ & $150 \pm 2$ \\
Brachial diastolic BP (mmHg) & $80 \pm 1$ & $81 \pm 1$ & $77 \pm 1^{* * *}$ \\
Brachial mean arterial pressure (mmHg) & $102 \pm 1$ & $103 \pm 1$ & $101 \pm 1$ \\
Brachial pulse pressure (mmHg) & $68 \pm 1$ & $65 \pm 1$ & $73 \pm 2^{* * *}$ \\
Aortic systolic BP (mmHg) & $137 \pm 1$ & $135 \pm 1$ & $140 \pm 2$ \\
Aortic diastolic BP (mmHg) & $82 \pm 1$ & $83 \pm 1$ & $79 \pm 1 * * *$ \\
Aortic mean arterial pressure (mmHg) & $105 \pm 1$ & $105 \pm 1$ & $104 \pm 1$ \\
Aortic pulse pressure (mmHg) & $55 \pm 1$ & $52 \pm 1$ & $61 \pm 2 * * *$ \\
Heart rate (beats/min) & $67 \pm 1$ & $67 \pm 1$ & $68 \pm 1$ \\
Augmentation (mmHg) & $16 \pm 1$ & $14 \pm 1$ & $20 \pm 1^{* * *}$ \\
Augmentation index (\%) & $27 \pm 1$ & $25 \pm 1$ & $32 \pm 1^{* * *}$ \\
\hline
\end{tabular}


Table 3 Univariate correlation coefficients for central pressure augmentation (adjusted for sex, age and heart rate) and maximum intima-media thickness (IMT) (adjusted for sex and age) vs various parameters in type 2 diabetic subjects

$f S$ fasting serum; $H b A_{1} c$ glycosylated haemoglobin; $n U$ nano unit

${ }^{*} p<0.05, * * p<0.01, * * * p<0.001$

for correlation

$N A$ not applicable; $n U A E R$

overnight urine albumin excretion rate

\begin{tabular}{|c|c|c|}
\hline & Central pressure augmentation $(\mathrm{mmHg})$ & Maximum IMT (mm) \\
\hline Duration of diabetes (years) & 0.133 & 0.024 \\
\hline \multicolumn{3}{|l|}{ Features of insulin resistance } \\
\hline Body mass index $(\mathrm{kg} / \mathrm{m} 2)$ & -0.110 & 0.111 \\
\hline Waist-to-hip ratio & -0.059 & -0.014 \\
\hline fS HDL cholesterol (mmol/l) & 0.087 & -0.100 \\
\hline fS triglycerides $(\mathrm{mmol} / \mathrm{l})$ & -0.013 & -0.100 \\
\hline \multicolumn{3}{|l|}{ Other parameters } \\
\hline fS glucose $(\mathrm{mmol} / \mathrm{l})$ & 0.073 & 0.029 \\
\hline $\mathrm{HbA}_{1} \mathrm{c}(\%)$ & -0.015 & -0.094 \\
\hline fS total cholesterol (mmol/l) & 0.021 & 0.044 \\
\hline fS LDL cholesterol (mmol/l) & -0.029 & 0.053 \\
\hline Albumin (nUAER) & $0.152 *$ & $0.165^{* *}$ \\
\hline Augmentation (mmHg) & NA & $0.230 * * *$ \\
\hline Augmentation index $(\%)$ & $0.614 * * *$ & $0.157 * *$ \\
\hline Maximum IMT (mm) & $0.230 * * *$ & NA \\
\hline
\end{tabular}

Structural changes in carotid arteries In a subgroup of 97 type 2 diabetic patients, the severity of carotid atherosclerosis was scored. Due to the smaller number of patients, the results were analysed jointly in men and women. There were no soft tissue changes in carotid arteries in 30 patients $(39 \%)$. Soft tissue changes were detected as follows: 32 patients with a score of $1(33 \%) ; 22$ patients with a score of $2(23 \%)$; and five patients with a score of $3(5 \%)$. Mineralisation changes were absent in 49 patients $(51 \%)$, whereas solitary mineralisations were found in 24 patients $(25 \%)$ and multiple mineralisation changes were found in 24 patients $(25 \%)$. No parameters other than IMT (ANOVA $p<0.001$, Fig. 1) differed significantly across the soft tissue categories. Although ANOVA results for differences in $\mathrm{AG}$ were not significant ( $p=$ 0.249 ), the difference between category 0 and categories 2 and 3 was almost significant $(p=0.088$ in a pairwise comparison). Several variables were significantly different across the mineralisation categories (Fig. 1). These included IMT (ANOVA $p<0.001$ ), aortic pulse pressure $(p<0.001)$, brachial pulse pressure $(p=0.001)$, aortic systolic pressure $(p=0.007)$, brachial systolic pressure $(p=$ $0.001)$, age $(p=0.010)$ and $\mathrm{AG}(p=0.016)$. Brachial and aortic diastolic pressures, lipids, $\mathrm{HbA}_{1} \mathrm{c}$, fasting glucose, smoking and diabetes duration did not differ between the categories.

\section{Discussion}

In the present study, a significant association was found between the ultrasonically determined carotid IMT and the variables $A G$ and $A I x$, measured using a tonometer and PWA, in type 2 diabetic patients. These associations were independent of age and sex, which are known to independently influence both arterial stiffness and IMT. Although women with type 2 diabetes had a reduced IMT, their AG and AIx values were increased when compared with those in men. None of the lipid parameters or markers of glycaemic control were correlated with IMT or arterial stiffness.

The present study did not aim to compare diabetic patients with non-diabetic subjects, but aimed to search for a potential relationship between arterial stiffness and carotid IMT, both surrogate markers of CVD risk. In a number of studies, increased arterial stiffness has been a consistent finding in type 2 diabetic patients [39-41], suggesting that arterial stiffness may contribute to accelerated atherosclerosis in type 2 diabetes. In the Strong Heart Study, arterial stiffness was measured using an ultrasound technique in 1,810 diabetic and 944 normoglycaemic American Indians [42]. The diabetic patients had significantly increased arterial stiffness. Moreover, diabetic status was independently associated with stiffness even after adjustment for age, sex, height, BMI, systolic blood pressure and use of antihypertensive medication [42]. In a smaller study by Brooks et al in which PWA was used, both $A G$ and AIx were increased in diabetic men but not in diabetic women [43]. Likewise, carotid IMT has consistently been shown to be greater in type 2 diabetic patients than in non-diabetic subjects [44-46]. In addition, IMT has correlated with the presence of CVD in type 2 diabetic patients [47].

To the best of our knowledge, the present study is the first to demonstrate in Caucasian type 2 diabetic patients that AG and carotid IMT are significantly inter-related. A similar relationship between IMT and AIx has previously been reported in non-diabetic subjects, but this difference disappeared after adjusting for age and sex [48]. In a Japanese study of 81 type 2 diabetic patients, AG correlated with IMT [49]. Furthermore, in a study by Taniwaki et al carotid IMT positively correlated with aortic pulse wave velocity, measured using B-mode ultrasound, in Japanese type 2 diabetic patients [50]. On the contrary, in an Indian study comprising 50 diabetic and 50 non-diabetic subjects, AIx correlated with IMT in the whole group and in the nondiabetic subset, but not in the diabetic subset [51], potentially due to lack of power. These inconsistent results 
a

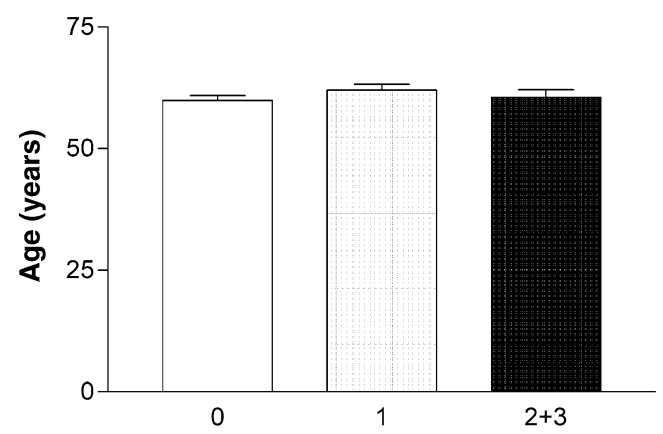

C

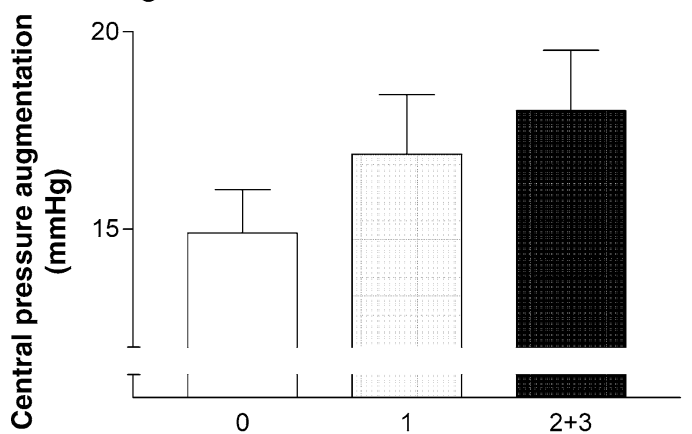

e

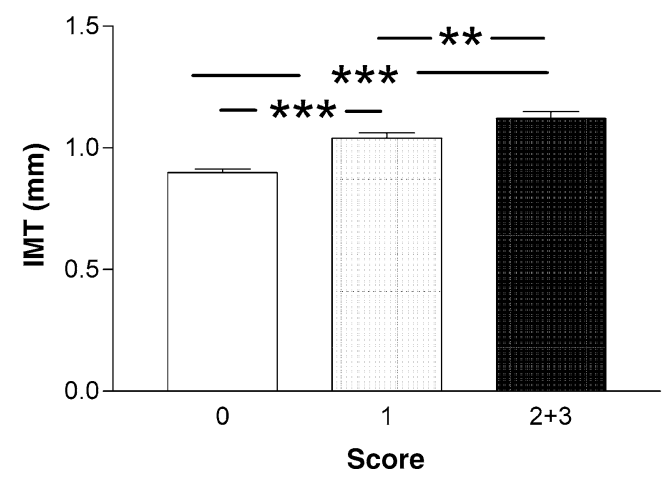

Fig. 1 Soft tissue scores (a, c, e) and mineralisation scores $(\mathbf{b}, \mathbf{d}, \mathbf{f})$ of carotid artery walls in type 2 diabetic patients $(n=97)$. For scoring system, see Subjects and Methods section. ANOVA: a NS; b

may also reflect differences in the prevalence of cardiovascular risk factors in the background population. In the aforementioned Indian study, the mean systolic and diastolic blood pressures were markedly lower $(126 / 81 \mathrm{mmHg}$ in the diabetic subset) than in our study. This definitely changes the relationship between IMT and AIx, as AIx is a function of central blood pressure.

Stiffening of the arteries occurs during ageing even in the absence of atherosclerosis [10]. In previous studies of non-diabetic subjects, AIx has been reported to correlate strongly with age $[14,52,53]$. This was not true for the type 2 diabetic patients in the present study. Our finding is consistent with the study by Nürnberger et al in which AIx was calculated along with the atherosclerosis scoring [54]. In subjects with established atherosclerotic disease, AIx did b

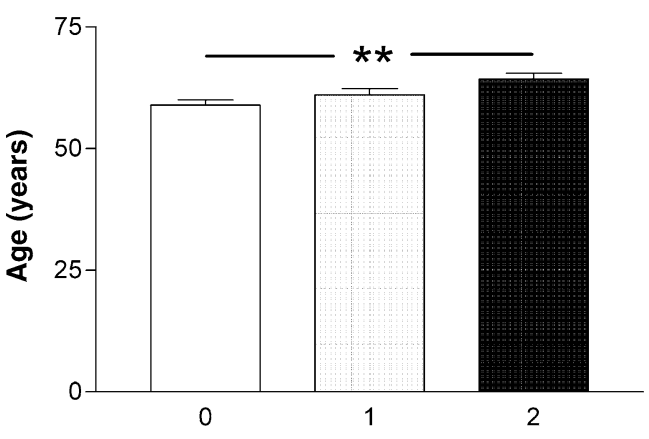

d

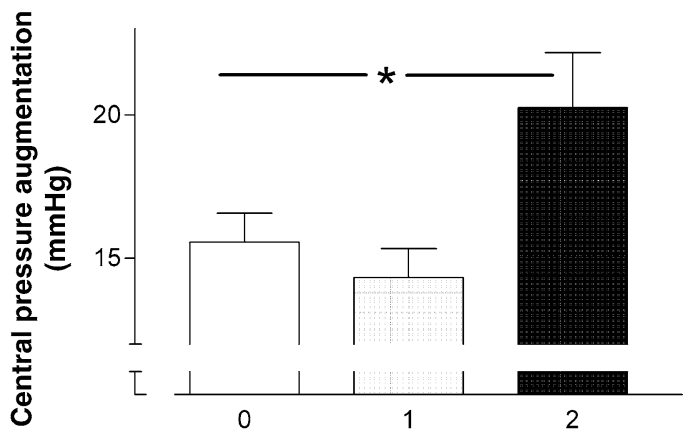

f

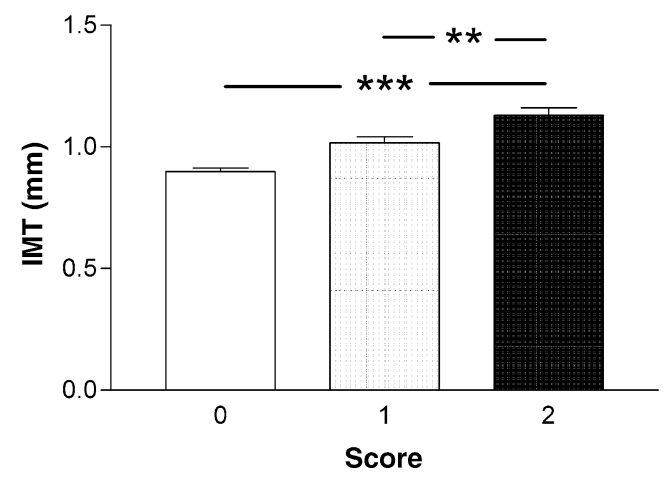

$p=0.010$; c. $p=0.249 ; \mathbf{d} p=0.016 ;$ e $p=0.001 ; \mathbf{f} p<0.001$ (comparison of scores across the categories). ${ }^{*} p<0.05 ; * * p<0.01 ; * * * p<0.001$ for pairwise comparisons

not correlate with age, in contrast to healthy subjects. While there was no correlation between AIx and age, AG was a significant correlate of age in men and women. Since AIx is calculated by dividing AG by pulse pressure, it is possible that accelerated vascular ageing in diabetes leads to an age-independent increase in pulse pressure, diluting the correlation. It is also worth noting that the age range of the present study was relatively narrow, thus potentially negating any correlation.

In the present study, large artery stiffness, as estimated from the increase in central wave reflection, i.e. the increase in AG, was increased in women compared with men. After age adjustment, the only correlates of IMT in multivariate analysis in men and women were AG and central systolic pressure. Type 2 diabetic women in partic- 
ular are at high risk of cardiovascular complications for reasons that are largely unknown $[55,56]$. One may speculate that increased AG increases central systolic pressure and attenuates diastolic filling of coronary arteries [57]. The increased AG in type 2 diabetic women compared with men in the present study suggests more profound deterioration of the central haemodynamics in type 2 diabetic women. IMT has repeatedly been reported to be reduced in women compared with men [58-60]. However, the risk of cardiovascular complications also increases proportionally in women with increasing IMT. In the Atherosclerosis Risk in Communities Study of 7,289 women and 5,552 men (follow-up 4-7 years), IMT predicted the incidence of coronary heart disease events in men and women [61]. Up to a mean IMT of $1 \mathrm{~mm}$, women had lower adjusted annual event rates than men, but above 1 $\mathrm{mm}$, the event rate in women was closer to that in men [31]. In the present study, mean IMT in female type 2 diabetic patients averaged $1 \mathrm{~mm}$, suggesting that they were at the same risk of cardiovascular complications as male type 2 diabetic patients.

We previously demonstrated that IMT in diabetic subjects is greater in patients with clinical cardiovascular disease than in those without, but this association disappeared after controlling for age, sex and smoking [62]. In the present study, there was no difference in $A G$ and AIx between diabetic patients with a history of clinical cardiovascular disease and those without. Thus, the relationship between central blood pressure indices and IMT does not transfer into a similar association with clinical cardiovascular disease. Acute cardiovascular events may be more a function of acute plaque rupture than of insidious vascular wall thickening and stiffening. The heterogeneity of our diabetic patients (ranging from newly diagnosed diabetic patients to subjects with a history of diabetes several decades long and cardiovascular disease) is, on the other hand, not ideal for detecting a relationship between arterial stiffening and cardiovascular events, as the patients are at very different points in the diabetic and atherosclerotic process. We did not find any significant correlation with IMT other than increased AG and AIx after adjustment for age and sex (Table 3). This is in line with the results of previous studies, in which the associations of traditional cardiovascular risk factors with IMT were weaker and less consistent in diabetic study cohorts than in non-diabetic cohorts [44, 45, 63-65]. In a subset of study patients, we observed that an increase in the mineralisation scores was associated with an increase in IMT, AG and peripheral and central systolic and pulse pressures. In accordance with this finding, Fukui et al recently showed that plaque score was associated with augmentation in Japanese type 2 diabetic patients [49].

Due to the cross-sectional nature of the present study, it cannot answer the question of causality between arterial stiffening and IMT. Prospective data on the predictive value of AG and AIx for cardiovascular morbidity and mortality are currently lacking in type 2 diabetic patients. A substudy of the ongoing FIELD investigation is expected to answer this question. The current cross-sectional data suggest that measures of central blood pressure and large artery stiffness may be superior to other conventional risk factors in determining the presence of vascular wall thickening in type 2 diabetes.

In conclusion, measures of central systolic pressure are correlated with carotid IMT, independently of age, and associated with arterial mineralisation changes in both men and women with type 2 diabetes. PWA provides a noninvasive rapid tool for measuring central haemodynamics. Prospective studies are needed to determine the value of PWA in predicting cardiovascular risk in diabetes.

Acknowledgements We would like to express our appreciation to the volunteers who participated in this study. We thank J. Tiikkainen and A. Malkki for reading the scans and $\mathrm{K}$. Ronkainen for data management. We also thank H. Hilden, R. Marjanen, H. Perttunen-Nio, V. Naatti and T. Silvennoinen for excellent technical assistance. This work was supported by grants from the Finnish Diabetes Association (E. Leinonen, M.-R. Taskinen), the Jenny and Antti Wihuri Fund (E. Leinonen), the Helsinki University Central Hospital Research Foundation (E. Leinonen, M.-R. Taskinen) and the Academy of Finland (J. Westerbacka, H. Yki-Järvinen) (all Helsinki, Finland). J. Westerbacka and E. Leinonen contributed equally to this work.

\section{References}

1. Fuller JH, Shipley MJ, Rose G, Jarrett RJ, Keen H (1983) Mortality from coronary heart disease and stroke in relation to degree of glycaemia: the Whitehall study. Br Med J 287:867870

2. Pyörälä K (1979) Relationship of glucose tolerance and plasma insulin in the incidence of coronary heart disease. Results from two population studies in Finland. Diabetes Care 2:131-141

3. Stamler J, Vaccaro O, Neaton JD, Wentworth D (1993) Diabetes, other risk factors, and 12-yr cardiovascular mortality for men screened in the Multiple Risk Factor Intervention Trial. Diabetes Care 16:434-444

4. Henry RM, Kostense PJ, Spijkerman AM et al (2003) Arterial stiffness increases with deteriorating glucose tolerance status: the Hoorn Study. Circulation 107:2089-2095

5. Kawasaki T, Sasayama S, Yagi S, Asakawa T, Hirai T (1987) Non-invasive assessment of the age related changes in stiffness of major branches of the human arteries. Cardiovasc Res 21:678687

6. Sonesson B, Hansen F, Stale H, Lanne T (1993) Compliance and diameter in the human abdominal aorta - the influence of age and sex. Eur J Vasc Surg 7:690-697

7. Hopkins KD, Lehmann ED, Gosling RG, Parker JR, Sonksen $\mathrm{PH}$ (1993) Biochemical correlates of aortic distensibility in vivo in normal subjects. Clin Sci (Colch) 84:593-597

8. Mohiaddin RH, Firmin DN, Longmore DB (1993) Age-related changes of human aortic flow wave velocity measured noninvasively by magnetic resonance imaging. J Appl Physiol 74: 492-497

9. O'Rourke MF, Blazek JV, Morreels CLJ, Krovetz LJ (1968) Pressure wave transmission along the human aorta. Changes with age and in arterial degenerative disease. Circ Res 23:567579

10. Avolio AP, Deng FQ, Li WQ et al (1985) Effects of aging on arterial distensibility in populations with high and low prevalence of hypertension: comparison between urban and rural communities in China. Circulation 71:202-210

11. O'Rourke MF, Gallagher DE (1996) Pulse wave analysis. J Hypertens 14:S147-S157 
12. Pauca AL, O'Rourke MF, Kon ND (2001) Prospective evaluation of a method for estimating ascending aortic pressure from the radial artery pressure waveform. Hypertension 38: 932-937

13. Nichols WW, O'Rourke MF (1998) McDonald's blood flow in arteries: theoretical, experimental and clinical principles. Arnold, London

14. Kelly R, Hayward C, Avolio A, O’Rourke M (1989) Noninvasive determination of age-related changes in the human arterial pulse. Circulation 80:1652-1659

15. Stefanadis C, Vlachopoulos C, Tsiamis E et al (1998) Unfavorable effects of passive smoking on aortic function in men. Ann Intern Med 128:426-434

16. Jonason T, Henrikssen E, Kangro T, Nilsson H, Vessby B, Ringqvist I (1997) Stiffness of the common carotid artery in healthy 50-year-old subjects. Clin Physiol 17:569-577

17. Kool MJ, Hoeks AP, Struijker BH, Reneman RS, Van Bortel LM (1993) Short- and long-term effects of smoking on arterial wall properties in habitual smokers. J Am Coll Cardiol 22: 1881-1886

18. Arnett DK, Boland LL, Evans GW et al (2000) Hypertension and arterial stiffness: the atherosclerosis risk in communities study. ARIC Investigators. Am J Hypertens 13:317-323

19. McVeigh GE, Burns DE, Finkelstein SM et al (1991) Reduced vascular compliance as a marker for essential hypertension. Am J Hypertens 4:245-251

20. Safar ME, Blacher J, Pannier B et al (2002) Central pulse pressure and mortality in end-stage renal disease. Hypertension 39:735-738

21. London GM, Blacher J, Pannier B et al (2001) Arterial wave reflections and survival in end-stage renal failure. Hypertension 38:434-438

22. Amann K, Tyralla K, Gross ML, Eifert T, Adamczak M, Ritz E (2003) Special characteristics of atherosclerosis in chronic renal failure. Clin Nephrol 60(Suppl 1):S13-S21

23. Weber T, Auer J, O'Rourke MF et al (2004) Arterial stiffness, wave reflections, and the risk of coronary artery disease. Circulation 109:184-189

24. Salonen R, Haapanen A, Salonen JT (1991) Measurement of intima-media thickness of common carotid arteries with highresolution B-mode ultrasonography: inter- and intra-observer variability. Ultrasound Med Biol 17:225-230

25. Veller MG, Fisher CM, Nicolaides AN et al (1993) Measurement of the ultrasonic intima-media complex thickness in normal subjects. J Vasc Surg 17:719-725

26. Belcaro G, Nicolaides AN, Laurora G et al (1996) Ultrasound morphology classification of the arterial wall and cardiovascular events in a 6-year follow-up study. Arterioscler Thromb Vasc Biol 16:851-856

27. Salonen R, Salonen JT (1995) Progression of carotid atherosclerosis and its determinants: a population-based ultrasonography study. Atherosclerosis 81:33-40

28. Craven TE, Ryu JE, Espeland MA et al (1990) Evaluation of the associations between carotid artery atherosclerosis and coronary artery stenosis. A case-control study. Circulation 82: $1230-1242$

29. Wofford JL, Kahl FR, Howard GR, McKinney WM, Toole JF, Crouse JR (1991) Relation of extent of extracranial carotid artery atherosclerosis as measured by B-mode ultrasound to the extent of coronary atherosclerosis. Arterioscler Thromb 11: 1786-1794

30. Salonen JT, Salonen R (1991) Ultrasonographically assessed carotid morphology and the risk of coronary heart disease. Arterioscler Thromb 11:1245-1249

31. Chambless LE, Heiss G, Folsom AR et al (1997) Association of coronary heart disease incidence with carotid arterial wall thickness and major risk factors: the Atherosclerosis Risk in Communities (ARIC) Study, 1987-1993. Am J Epidemiol 146: 483-494

32. Pauca AL, Wallenhaupt SL, Kon ND, Tucker WY (1992) Does radial artery pressure accurately reflect aortic pressure? Chest 102:1193-1198
33. Leskinen Y, Lehtimaki T, Loimaala A et al (2003) Carotid atherosclerosis in chronic renal failure - the central role of increased plaque burden. Atherosclerosis 171:295-302

34. Arad Y, Spadaro LA, Roth M et al (1998) Correlations between vascular calcification and atherosclerosis: a comparative electron beam CT study of the coronary and carotid arteries. J Comput Assist Tomogr 22:207-211

35. Ylitalo K, Syvanne M, Salonen R, Nuotio I, Taskinen MR, Salonen JT (2002) Carotid artery intima-media thickness in Finnish families with familial combined hyperlipidemia. Atherosclerosis 162:171-178

36. Westerbacka J, Wilkinson I, Cockcroft J, Utriainen T, Vehkavaara S, Yki-Järvinen H (1999) Diminished wave reflection in the aorta: a novel physiological action of insulin on large blood vessels. Hypertension 33:1118-1122

37. Karamanoglu M, O’Rourke MF, Avolio AP, Kelly RP (1993) An analysis of the relationship between central aortic and peripheral upper limb pressure waves in man. Eur Heart J $14: 160-167$

38. Wilkinson IB, MacCallum H, Flint L, Cockcroft JR, Newby DE, Webb DJ (2000) The influence of heart rate on augmentation index and central arterial pressure in humans. J Physiol 525(Pt 1):263-270

39. Glasser SP, Arnett DK, McVeigh GE et al (1997) Vascular compliance and cardiovascular disease: a risk factor or a marker? Am J Hypertens 10:1175-1189

40. Lehmann ED, Riley WA, Clarkson P, Gosling RG (1999) Noninvasive assessment of cardiovascular disease in diabetes mellitus. Lancet 350:14-19

41. McVeigh GE, Brennan G, Hayes R, Cohn J, Finkelstein S, Johnston D (1993) Vascular abnormalities in non-insulindependent diabetes mellitus identified by arterial waveform analysis. Am J Med 95:424-430

42. Devereux RB, Roman MJ, Paranicas M et al (2000) Impact of diabetes on cardiac structure and function: the strong heart study. Circulation 101:2271-2276

43. Brooks BA, Molyneaux LM, Yue DK (2001) Augmentation of central arterial pressure in type 2 diabetes. Diabet Med 18:374 380

44. Mohan V, Ravikumar R, Shanthi RS, Deepa R (2000) Intimal medial thickness of the carotid artery in South Indian diabetic and non-diabetic subjects: the Chennai Urban Population Study (CUPS). Diabetologia 43:494-499

45. Temelkova-Kurktschiev TS, Koehler C, Leonhardt W et al (1999) Increased intimal-medial thickness in newly detected type 2 diabetes: risk factors. Diabetes Care 22:333-338

46. Haffner SM, Agostino RDJ, Saad MF et al (2000) Carotid artery atherosclerosis in type- 2 diabetic and nondiabetic subjects with and without symptomatic coronary artery disease (The Insulin Resistance Atherosclerosis Study). Am J Cardiol 85:1395-1400

47. Mitsuhashi N, Onuma T, Kubo S, Takayanagi N, Honda M, Kawamori R (2002) Coronary artery disease and carotid artery intima-media thickness in Japanese type 2 diabetic patients. Diabetes Care 25:1308-1312

48. Saba PS, Roman MJ, Pini R, Spitzer M, Ganau A, Devereux RB (1993) Relation of arterial pressure waveform to left ventricular and carotid anatomy in normotensive subjects. J Am Coll Cardiol 22:1873-1880

49. Fukui M, Kitagawa Y, Nakamura N et al (2003) Augmentation of central arterial pressure as a marker of atherosclerosis in patients with type 2 diabetes. Diabetes Res Clin Pract 59:153161

50. Taniwaki H, Kawagishi T, Emoto M et al (1999) Correlation between the intima-media thickness of the carotid artery and aortic pulse-wave velocity in patients with type 2 diabetes. Vessel wall properties in type 2 diabetes. Diabetes Care 22: $1851-1857$ 
51. Ravikumar R, Deepa R, Shanthirani C, Mohan V (2002) Comparison of carotid intima-media thickness, arterial stiffness, and brachial artery flow mediated dilatation in diabetic and nondiabetic subjects (The Chennai Urban Population Study [CUPS-9]). Am J Cardiol 90:702-707

52. Westerbacka J, Seppälä-Lindroos A, Yki-Järvinen H (2001) Resistance to acute insulin induced decreases in large artery stiffness accompanies the insulin resistance syndrome. J Clin Endocrinol Metab 86:5262-5268

53. Wilkinson IB, Prasad K, Hall IR et al (2002) Increased central pulse pressure and augmentation index in subjects with hypercholesterolemia. J Am Coll Cardiol 39:1005-1011

54. Nurnberger J, Keflioglu-Scheiber A, Opazo SA, Wenzel RR, Philipp T, Schafers RF (2002) Augmentation index is associated with cardiovascular risk. J Hypertens 20:2407-2414

55. Kannel WB, Abbott RD (1987) Incidence and prognosis of myocardial infraction in women: the Framingham study. In: Eaker ED, Packard B, Wenger NK, Clarkson TB, Tyroler HA (eds) Coronary heart disease in women. Haymarket-Doyma, New York, pp 208-214

56. Barrett-Connor EL, Cohn BA, Wingard DL, Edelstein SL (1991) Why is diabetes mellitus a stronger risk factor for fatal ischemic heart disease in women than in men? The Rancho Bernardo Study. JAMA 265:627-631

57. O'Rourke MF, Kelly RP (1993) Wave reflection in the systemic circulation and its implications in ventricular function. J Hypertens 11:327-337

58. Urbina EM, Srinivasan SR, Tang R, Bond MG, Kieltyka L, Berenson GS (2002) Impact of multiple coronary risk factors on the intima-media thickness of different segments of carotid artery in healthy young adults (The Bogalusa Heart Study). Am J Cardiol 90:953-958
59. Riley WA, Evans GW, Sharrett AR, Burke GL, Barnes RW (1997) Variation of common carotid artery elasticity with intimal-medial thickness: the ARIC Study. Atherosclerosis risk in communities. Ultrasound Med Biol 23:157-164

60. Lekakis JP, Papamichael CM, Cimponeriu AT et al (2000) Atherosclerotic changes of extracoronary arteries are associated with the extent of coronary atherosclerosis. Am J Cardiol 85: 949-952

61. Chambless LE, Heiss G, Folsom AR et al (1997) Association of coronary heart disease incidence with carotid arterial wall thickness and major risk factors: the Atherosclerosis Risk in Communities (ARIC) Study, 1987-1993. Am J Epidemiol 146:483-494

62. Leinonen ES, Hiukka A, Hurt-Camejo E et al (2004) Lowgrade inflammation, endothelial activation and carotid intimamedia thickness in type 2 diabetes. J Intern Med 256:119-127

63. Yamasaki Y, Kawamori R, Matsushima H et al (1995) Asymptomatic hyperglycaemia is associated with increased intimal plus medial thickness of the carotid artery. Diabetologia 38:585591

64. Goff DCJ, D'Agostino RBJ, Haffner SM, Saad MF, Wagenknecht LE (2000) Lipoprotein concentrations and carotid atherosclerosis by diabetes status: results from the insulin resistance atherosclerosis study. Diabetes Care 23:1006-1011

65. Kong C, Elatrozy T, Anyaoku V, Robinson S, Richmond W, Elkeles RS (2000) Insulin resistance, cardiovascular risk factors and ultrasonically measured early arterial disease in normotensive type 2 diabetic subjects. Diabetes Metab Res Rev 16:448453 